\title{
Telemedizin
}

\section{Gute Ansätze, noch viele Unklarheiten}

Liebe Leserinnen und Leser,

definiert man Telemedizin als Kommunikation zwischen Patient und Arzt sowie unter Ärzten, hat diese Disziplin bereits eine lange Geschichte. Dann standen am Beginn die Versendung einer Urinprobe mit der Postkutsche vom Patienten zum Arzt oder eines histologischen Präparates per Post vom Arzt an den Pathologen. Heute verdeutlicht die ärztliche Betreuung von Raumfahrern im Weltall, wie eindrucksvoll und rasant die technischen Entwicklungen auch das Spektrum der audiovisuellen Kommunikation für die Medizin erweitert haben.

Das Präfix „Tele-,, haben sich inzwischen alle Fachdisziplinen von der Chirurgie über die Psychiatrie und die Prävention bis zur Therapie angeeignet. Die Techniken und Methoden zeichnen sich durch eine ungeheure Vielfalt aus. Zum Standard gehört heute, wenn Kliniken ohne Pathologen histologische Präparate (z.B. Schnellschnitte) oder schwer deutbare Röntgenbilder dem entfernten Pathologen oder Radiologen zuspielen, um sie umgehend live zu diskutieren und zu interpretieren.

\section{Telekonsile in Schlaganfall-Projekt}

Ein eindrucksvolles Beispiel: Bei der Betreuung von Patienten mit akutem Schlaganfall können Ärzte in regionalen Kliniken rund um die Uhr mit zwei akademischen Schlaganfallzentren kommunizieren (TEMPiS*-Projekt). Schon in der Notaufnahme können die Experten per Videoschaltung die Patienten in den entfernten Kliniken direkt befragen und zusammen mit dem Arzt vor Ort neurologisch untersuchen, die Tomografiebilder beurteilen und innerhalb kurzer Zeit über die Indikation zur Lysetherapie entscheiden. Seit der Gründung im Jahr 2003 erfolgten über 20000 derartige Telekonsile. Das Projekt umfasst heute 15 Kooperationskliniken, in denen im Jahr 2009 über 6000 akute Schlaganfälle und TIA's behandelt wurden (Müller-Baba 2011). V.a. wurde bereits ein Wirksamkeitsnachweis für Patienten der kooperierenden im Vergleich zu unbeteiligten Kliniken nach den Kriterien „Mortalität", „institutionalisierter Pflegedürftigkeit" und „schwerer Hilfsbedürftigkeit" erbracht.

\section{Nicht nur Abrechnungsfragen sind ungeklärt}

In diesem Heft wird über den gegenwärtigen Stand einer Teildisziplin der Telemedizin, dem Telemonitoring von Blutzucker, Blutdruck und Symptomen kardiovaskulärer Erkrankungen bei Diabetikern berichtet (s. S. 40). Man erwartet, dass durch die engmaschige Überwachung der Patienten unzureichende Stoffwechseleinstellungen, klinische Verschlechterungen und drohende Komplikationen früher erkannt und behandelt, stationäre Aufnahmen vermieden, die Behandlungsqualität optimiert und die Kosten gesenkt werden. Viele grundsätzliche Probleme - nicht nur auf dem Gebiet der Diabetologie - sind derzeit aber noch ungelöst.

1. Trotz vieler, überwiegend aber kleiner und methodisch unzureichender Studien ist unklar, ob die genannten Erwartungen erfüllt werden.

2. Alle telemedizinischen Anwendungen werden den Nachweis von Wirksamkeit, Zweckmäßigkeit und Wirtschaftlichkeit erbringen müssen, bevor sie als abrechnungsfähige Leistungen in die Gebührenordnung übernommen werden. Dabei wird auch geprüft werden, ob einzelne telemedizinische Techniken eine eigene Abrechnungsziffer oder als bereits bestehende, nun aber telemedizinisch assistierte Methoden eine höhere Punktzahl erhalten.

3. Trotz des teils erheblichen personellen, apparativen und technischen Aufwands wird erwartet, dass telemedizinische Techniken die Gesundheitskosten senken, obwohl es bisher durch neue Methoden meist Kostensteigerungen gab.

4. In der bisherigen Debatte um die Telemedizin sind standespolitisch bedeutsame Fragen zu wenig diskutiert worden. So verlangt das Telemonitoring bei bestimmten Krankheiten (z.B. Risikopatienten für bedrohliche Herzrhythmusstörungen) eine kontinuierliche Bewertung der eingehenden Daten und adäquate Sofortreaktionen rund um die Uhr, die nur von spezialisierten Zentren geleistet werden können. Wird bei weniger akut Bedrohlichem wie Diabetes und Herzinsuffizienz der niedergelassene Arzt die Daten seiner telemetrisch überwachten Patienten zeitgerecht sichten und beurteilen, und bei Bedarf auch adäquat, d.h. durch Telefonat, Hausbesuch oder Einweisung reagieren können? Oder muss er die Patienten abgeben?

5. Die Telemedizin erweitert das Arzt-PatientenVerhältnis um einen nicht personalen Aspekt. Standespolitische und komplexe juristische Probleme (z.B. die Haftung für eingehende, nicht adäquat bearbeitete Befundmeldungen) sind bisher unzureichend gelöst.

Ihr Heinrich Holzgreve

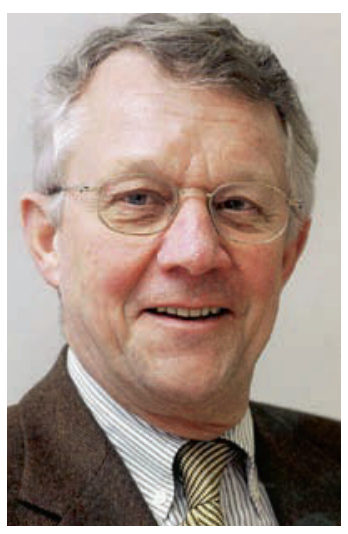

Prof. Dr. med. Heinrich Holzgreve, Internist in München und Schriftleiter der Info Diabetologie
* Telemedizinisches Projekt zur integrierten Schlaganfallversorgung in der Region Süd-OstBayern

http://www.tempis.de/ 